\title{
Aggregation of F-Actin in Olfactory Glomeruli: a Common Feature of Glomeruli Across Phyla
}

\section{Wolfgang Rössler, Joško Kuduz, Friedrich W. Schürmann and Detlev Schild}

\author{
Physiologisches Institut, Universität Göttingen, Humboldtallee 23, 37073 Göttingen, Germany
}

Correspondence to be sent to (current address): Wolfgang Rössler, Biozentrum der Universität, Zoologie II, Am Hubland, 97074 Würzburg, Germany. e-mail: roessler@biozentrum.uni-wuerzburg.de

\begin{abstract}
Using fluorophore-conjugated phalloidin, we show that filamentous (F)-actin is strongly aggregated in olfactory glomeruli within primary olfactory centers of vertebrates and insects. Our comparative study demonstrates that aggregation of F-actin is a common feature of glomeruli across phyla, and is independent of glomerular architecture and/or the presence or absence of cellular borders around glomeruli formed by neurons or glial cells. The distribution of F-actin in axonal and dendritic compartments within glomeruli, however, appears different between vertebrates and insects. The potential role of the actin-based cytoskeleton in synaptic and structural plasticity within glomeruli is discussed.
\end{abstract}

\section{Introduction}

Primary olfactory centers in the brains of vertebrates as well as most advanced invertebrates typically are compartmentalized in spheroidal areas of dense synaptic neuropil called olfactory glomeruli. Recent evidence suggests that glomeruli represent functional units in olfactory information processing (Hildebrand and Shepherd, 1997; Valverde, 1999; Xu et al., 2000). In vertebrates, glomerular synapses undergo a lifelong turnover, resulting from continuous regeneration of axons from olfactory receptor neurons (ORNs) that are constantly replaced by basal cells in the olfactory epithelium (Wolff and Missler, 1992; Farbman, 1994; Astic and Saucier, 2001). In insects, individual glomeruli exhibit a high degree of plasticity during postembryonic development, as well as activity-dependent structural changes during adult life (Winnington et al., 1996; Sigg et al., 1997; Rössler et al., 1999a). Thus, the glomerular synaptic neuropil in both vertebrates and insects is characterized by a high degree of synaptic and structural plasticity. To further understand the organization and function of glomeruli, a comparative approach to structural, molecular and physiological properties of olfactory glomeruli is particularly promising, and should lead to the discovery of the most essential features of olfactory glomeruli.

Despite similarities in some general features, anatomical and cellular attributes of individual glomeruli and the organization of the entire array of glomeruli can differ markedly across species. For example, in mammals and insects, glomeruli were shown to be very distinct and clearly separated by glomerular borders formed by periglomerular neurons and/or glial cells, which also play an important role during development (Tolbert and Oland, 1990; Treolar et al., 1999). In other species, especially in lower vertebrates, regular cellular borders around glomeruli are absent, and the glomerular synaptic neuropil appears much less defined compared with the conditions in mammals (Byrd and Burd, 1991; Nezlin and Schild, 2000). Despite these obvious differences in glomerular organization, the olfactory system of lower vertebrates, e.g. fish, was shown to perform with high sensitivity in olfactory discrimination tasks and expresses a remarkable regenerative capacity (Zippel, 2000).

Here we show that glomeruli in the primary olfactory centers of various species of vertebrates and insects are characterized by strong aggregation of filamentous $(F)$ actin, independent of the presence or absence of periglomerular borders and differences in the general organization of the glomerular array. The results show that fluorophoreconjugated phalloidin reliably labels glomeruli in a broad range of animal species, allowing distinct mapping of glomeruli even in species that show a very loose or no organization of glomeruli in classical histological preparations. We conclude that distinct aggregation of F-actin in olfactory glomeruli is a fundamental feature of glomeruli across phyla and could play an important role in synaptic and structural plasticity within primary olfactory centers.

\section{Materials and methods}

\section{Animals and tissue preparation}

We used the following species: Xenopus laevis [tadpoles, stages 49-54 (Nieuwkoop and Faber, 1956)], axolotl 
(Ambystoma mexicanum, from Dr D.L. Meyer, Göttingen, Germany), goldfish (Carassius auratus, kindly provided by Dr H.P. Zippel, Göttingen, Germany), tobacco hawkmoth (Manduca sexta, kindly provided by Dr J. Schachtner, Marburg, Germany), giant silkmoth (Antherea polyphemus, kindly provided by Dr E. Kaissling, Seewiesen, Germany), honeybee (Apis mellifera, kindly provided by Dr J. Rybak, Würzburg, Germany), three species of ant (kindly provided by Drs. J. Liebig and B. Hölldobler, Würzburg, Germany) and cricket (Gryllus bimaculatus, laboratory colony, Dr Schürmann, Göttingen) for the experiments. Dr M. Rickmann (Göttingen, Germany) provided a perfusion-fixed rat brain and Dr O. Stiedl (Göttingen) a perfusion-fixed mouse brain (strain C57 BL/6NCrlBR). All animals were anesthetized and killed using appropriate methods. After dissection, the brains were immediately transferred to cold solution of $4 \%$ formaldehyde in phosphate buffered saline (PBS), pH 7.2 or 7.4, and fixed over night at $4^{\circ} \mathrm{C}$. Brains were washed at least three times in PBS, embedded in 5\% low melting point agarose (Agarose type II, no. 210-815; Amreso, Inc., Solon, OH), sectioned at $50-300 \mu \mathrm{m}$ on a Vibratome (Leica VT 1000S) or processed as whole mounts and sectioned after the staining procedures.

\section{Labeling with phalloidin, immunocytochemistry and nuclear staining with propidium iodide}

Sections were stained with Alexa Fluor 488 phalloidin (A-12379, Molecular Probes, Eugene, OR). We obtained the best results when sections were preincubated $(1 \mathrm{~h}$ at room temperature) in $0.1 \mathrm{M}$ PBS with $0.2 \%$ Triton-X and $2 \%$ normal goat serum (NGS; no. 191356, ICN Biomedicals $\mathrm{GmbH}$, Orsay, France). Preincubation with Triton and NGS was not always essential and should be tested for any particular species. We observed a reduction in background after preincubating with NGS. Free-floating agarose sections or whole brains were incubated in 0.2 units of Alexa Fluor 488 phalloidin $(1 \mu 1$ methanolic stock solution in $200 \mu \mathrm{PBS}$ with $2 \% \mathrm{NGS}$ ) initially for $2-4 \mathrm{~h}$ at room temperature (RT), and subsequently overnight or for 2 days at $4^{\circ} \mathrm{C}$ on a shaker.

Some preparations were double-labeled with antibodies to synaptic proteins. Antibodies to Synapsin (monoclonal, derived from mouse) were kindly provided by Dr E. Buchner (Biozentrum, University of Würzburg, Germany) (Klagges et al., 1996), antibodies to synaptophysin (polyclonal G113/p38 frog, derived from rabbit) were generously supplied by Dr R. Jahn (Max Planck Institute for Biophysical Chemistry, Göttingen, Germany) (Jahn et al. 1985). Sections were washed in PBS with $0.2 \%$ Triton X-100 (PBST), and nonspecific binding was blocked with $2 \%$ NGS in PBST for $1 \mathrm{~h}$ at RT. Then, the tissue was incubated overnight at $4{ }^{\circ} \mathrm{C}$ with anti-Synaptophysin (1:1000) or anti-Synapsin (1:50), diluted in PBS with $2 \%$ NGS, together with phalloidin (see above). Sections were subsequently washed with PBS, and Alexa 488- or 546-conjugated goat anti rabbit or anti mouse secondary antibodies (A 11008 and A 11003; Molecular Probes) were applied at a dilution of $1: 250$ in $1 \%$ NGS/PBS for $2 \mathrm{~h}$ at RT. The secondary antibodies were washed in five changes of PBS.

To stain glial and neuronal cell nuclei, sections (or whole mounts) were incubated for $15-30 \mathrm{~min}$ in $25 \mu \mathrm{g} / \mathrm{ml}$ propidium iodide (P-1304, Molecular Probes) in PBS. Finally, preparations were washed in at least five changes of PBS, transferred in $60 \%$ glycerol/PBS for at least $1 \mathrm{~h}$, and finally mounted on slides for confocal microscopy in $80 \%$ glycerol/PBS.

\section{Laser scanning confocal microscopy}

Preparations were viewed using a laser-scanning confocal microscope (Zeiss LSM 510) attached to an inverted microscope (Zeiss Axiovert 100M). A $15 \mathrm{~mW}$ argon ion and a $1 \mathrm{~mW}$ helium/neon laser were used as light sources. Single optical sections as well as series of sections were imaged at intervals of $0.5-5 \mu \mathrm{m}$ through the depth of the thick sections. They were saved as single optical images or three-dimensional stacks. Two-dimensional projections were generated for each channel and merged with the use of pseudocolors. Where needed, the digitized images were modified only to enhance contrast, to merge images from consecutive sections of the same preparation, or to form montages of images from adjacent regions. Image processing and labeling of figures were performed with one or more of the following programs: Zeiss imaging software

Figure 1 Distribution of phalloidin labeled F-actin in the olfactory bulb (OB) of vertebrates. (A, B) Xenopus laevis tadpole (stage 53): sagittal section of the main $\mathrm{OB}(\mathrm{A})$ and higher magnification of a horizontal section (B). Double staining with phalloidin (green) and propidium iodide (red). Note that individual glomeruli are not separated by periglomerular cellular borders but show a distinct aggregation of F-actin. The arrow in (A) indicates very small glomeruli in the dorsal OB. (C, D) Xenopus laevis tadpole (stage 53): double labeling of the accessory OB with phalloidin (C, green) and anti-synaptophysin antibody (D, red). Same magnification as the main OB shown in (B). (E, F) Ambystoma mexicanum (axolotl): double labeling of the OB with phalloidin (green) and prodpidium iodide (red). Section showing the glomerular layer of the OB with distinct glomeruli of variable shape and size. (G) Carassius auratus (goldfish): glomerular layer in the OB labeled with phalloidin (green) and prodpidium iodide (red). (H) Rat: glomeruli in the main OB (Phalloidin in green, prodidium iodide in red). (J, K) Rat: double staining of glomeruli with phalloidin (green) and anti-synapsin (red). (L) Mouse: section of the OB labeled with phalloidin only showing bright labeling in the glomeruli and homogeneous, lighter staining in the external and internal plexiform layers (EPL, IPL). (M) Xenopus laevis: glomeruli at high magnification stained with phalloidin (green) and anti-synaptophysin antibody (red). Single optical section (0.5 $\mu \mathrm{m}$ thickness). The arrows indicate synaptophysin-IR spots clustered around phalloidin-labeled structures indicating predominantly postsynaptic localization of F-actin. Scale bars: A = $200 \mu \mathrm{m}, \mathrm{B}=100 \mu \mathrm{m}, \mathrm{C}, \mathrm{D}=100 \mu \mathrm{m}, \mathrm{E}=100 \mu \mathrm{m}, \mathrm{F}=20 \mu \mathrm{m}, \mathrm{G}=100 \mu \mathrm{m}, \mathrm{H}=100 \mu \mathrm{m}, \mathrm{J}-\mathrm{L}=100 \mu \mathrm{m}, \mathrm{M}=10 \mu \mathrm{m}$. EPL, external plexiform layer; GL, glomerular layer; GCL, granule cell layer; IPL, internal plexiform layer; $\mathrm{MCL}$, mitral cell layer; $\mathrm{NL}$, nerve layer; ON, olfactory nerve; $\mathrm{V}$, ventricle. 

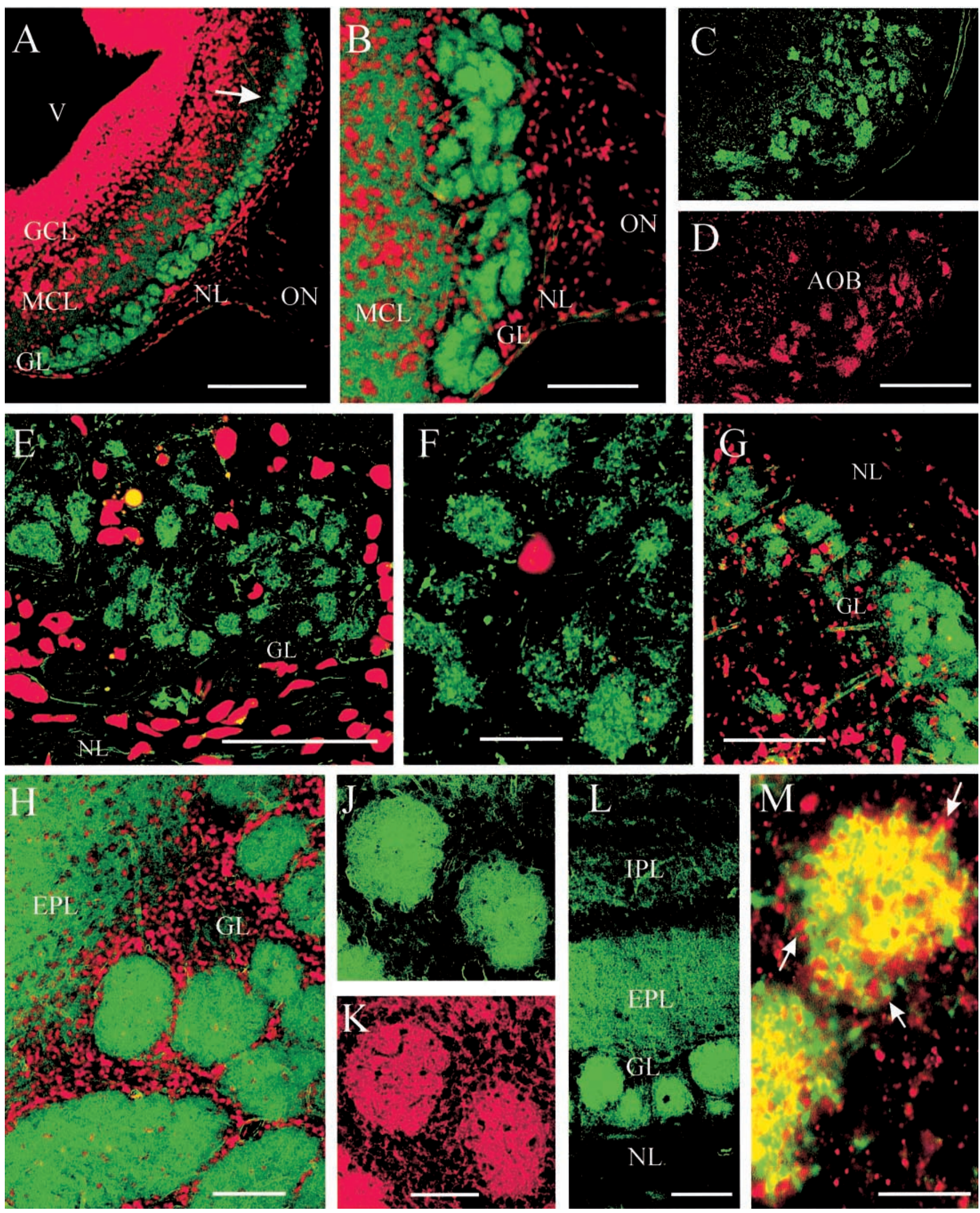
(Zeiss, Göttingen, Germany), Corel Photopaint and Corel Draw (Corel Corporation, Ottawa, Ontario, Canada).

\section{Results}

\section{Glomerular aggregation of F-actin: comparison among species}

In all species we investigated, olfactory glomeruli became brightly labeled after phalloidin treatment (Figures 1 and 2). The concentration of phalloidin within glomeruli was high compared with the surrounding tissue. In lower and higher vertebrates and in all insects we investigated, staining was present across the entire volume of individual glomeruli. Only in some cases did we observe differences in the staining intensity of individual glomeruli (see below). Labeling of cell nuclei with propidium iodide demonstrates that glomeruli in the olfactory bulb (OB) of Xenopus laevis (Figure 1A,B) and Ambystoma mexicanum (Figure 1E,F) are not surrounded by a regular border of periglomerular somata of neurons or glial cells [for $X$. laevis, see also (Nezlin and Schild, 2000)]. The distribution of phalloidin, even in these cases, shows a distinct aggregation of F-actin in a glomerular pattern. In both species, classical histological techniques show either a very loose glomerular organization [Xenopus (Byrd and Burd, 1991)] or the complete absence of a glomerular pattern [Ambystoma (D.L. Meyer, unpublished data)]. F-actin aggregations in Ambystoma were more separated compared with Xenopus, but glomeruli were easy to detect in both species (Figure 1A,B,E,F). In $X$. laevis, more homogeneous labeling at lower intensity was present in the plexiform layer of the $\mathrm{OB}$ (Figure 1A,B). In the accessory olfactory bulb of Xenopus laevis, too, distinct glomerular aggregation of F-actin was present, but glomeruli were significantly smaller and more irregular in shape (Figure 1C). In the OB of the goldfish (Figure 1G), glomeruli became clearly labeled, but in some areas of the OB glomeruli were less distinct compared with the conditions in amphibians, and especially compared with the mammalian $\mathrm{OB}$. In the rat $\mathrm{OB}$, the entire volume of glomeruli was brightly labeled up to the border formed by periglomerular neurons, and homogeneous staining was present in the plexiform layer (Figure 1H). In all vertebrates, blood vessels were stained with phalloidin, but were easy to distinguish from neuronal structures. The picture was very similar in the mouse $\mathrm{OB}$, where the staining intensity was highest in the glomeruli and showed decreasing levels in the external and internal plexiform layers (Figure 1L). Taken together, phalloidin labeling of olfactory glomeruli showed great similarities across various species of lower and higher vertebrates, despite significant differences in glomerular shape and volume, and independent of the presence or absence of cellular borders around glomeruli.

In insects, too, phalloidin labeling indicates high concentration of $\mathrm{F}$-actin within glomeruli (Figure 2). As in vertebrates, the entire volume of all glomeruli was filled with labeled neuronal branches, and staining was absent from interglomerular spaces. In females of the moth Manduca sexta, individual identified glomeruli, like the two large, lateral female-specific glomeruli (LFGs) at the entrance of the antennal lobe (AL), could easily be recognized [Figure 2A; for comparison see (Rössler et al., 1998, 1999a)]. The same was true for the large glomeruli of the macroglomerular complex in the AL of male moths and for other identified glomeruli (data not shown). In the silkmoth Antherea polyphemus, the pattern was very similar to the conditions in $M$. sexta (data not shown). In both species of moths, a prominent border formed by glial somata surrounds individual glomeruli [Figure 2C; see also (Tolbert and Hildebrand, 1981; Oland and Tolbert, 1996)]. Phalloidin labeling was completely absent from the glial cells forming the border (Figure 2C) and from peripheral glial cells within the antennal (olfactory) nerve (Figure 2B).

In two examples of hymenopteran insects, the honeybee (Apis mellifera) and the ponerine ant (Harpegnathos saltator), glomeruli were not surrounded by glial somata, but also in these cases phalloidin labeling was concentrated in glomeruli and absent from interglomerular spaces (Figure 2D,E,H). The example of the ALs in the ant $H$. saltator demonstrates that projections from stacks of confocal images allow detailed analyses of the organization and bilateral symmetry of the entire glomerular array, even in small species of insects (Figure $2 \mathrm{H}$ ).

Figure 2 Distribution of phalloidin labeled F-actin in the antennal lobe (AL) of insects. (A-C) Manduca sexta: (A) antennal lobe of a female double labeled with phalloidin (green) and propidium iodide (red). Two large female-specific glomeruli are visible at the entrance of the AL. (B) Antennal nerve entering the AL containing glial nuclei in the sorting zone (SZ) and lightly stained olfactory-receptor axons. (C) Single glomerulus with a typical border formed by glial cell bodies. (D-G) Apis mellifera (honeybee): (D, E) antennal lobe and high magnification of individual glomeruli labeled with phallodin (green) and propdium iodide (red). Note the absence of phalloidin in interglomerular spaces despite the lack of glial cell bodies. (F, G) Group of glomeruli double labeled with phalloidin (green) and anti-synapsin antibody (red). Note that axonal tracts are labeled with phallodin but not with anti-synapsin. (H) Harpegnathos saltator (ponerine ant): projection of a stack of confocal images (72 $\mu \mathrm{m}$ thickness) of the antennal lobes showing almost symmetrical arrangement of glomeruli on both sides. In this species, glomeruli in the frontal and medial areas of the ALs became most intensely labeled. The inset shows an individual glomerulus at high magnification. (J) Gryllus bimaculatus (cricket): single optical section of an individual glomerulus at high magnification. Projection-neuron dendrites labeled with rhodamin-coupled dextrans (red) and phalloidin staining (green) show very little overlap indicating predominant localization of F-actin in axonal compartments. Scale bars: $A=200 \mu \mathrm{m}, \mathrm{B}=50 \mu \mathrm{m}, \mathrm{C}=20 \mu \mathrm{m}, \mathrm{D}=100 \mu \mathrm{m}, \mathrm{E}=20 \mu \mathrm{m}, \mathrm{F}, \mathrm{G}=20 \mu \mathrm{m}, \mathrm{H}=100 \mu \mathrm{m}$ (inset in H=10 $\mu \mathrm{m}$ ), J=20 $\mu \mathrm{m}$. $\mathrm{AL}$, antennal lobe; $\mathrm{AN}$, antennal nerve; LC, lateral cluster of somata of AL neurons; MC, medial cluster of somata of $\mathrm{AL}$ neurons; $\mathrm{SZ}$, glia-rich axon sorting zone. 

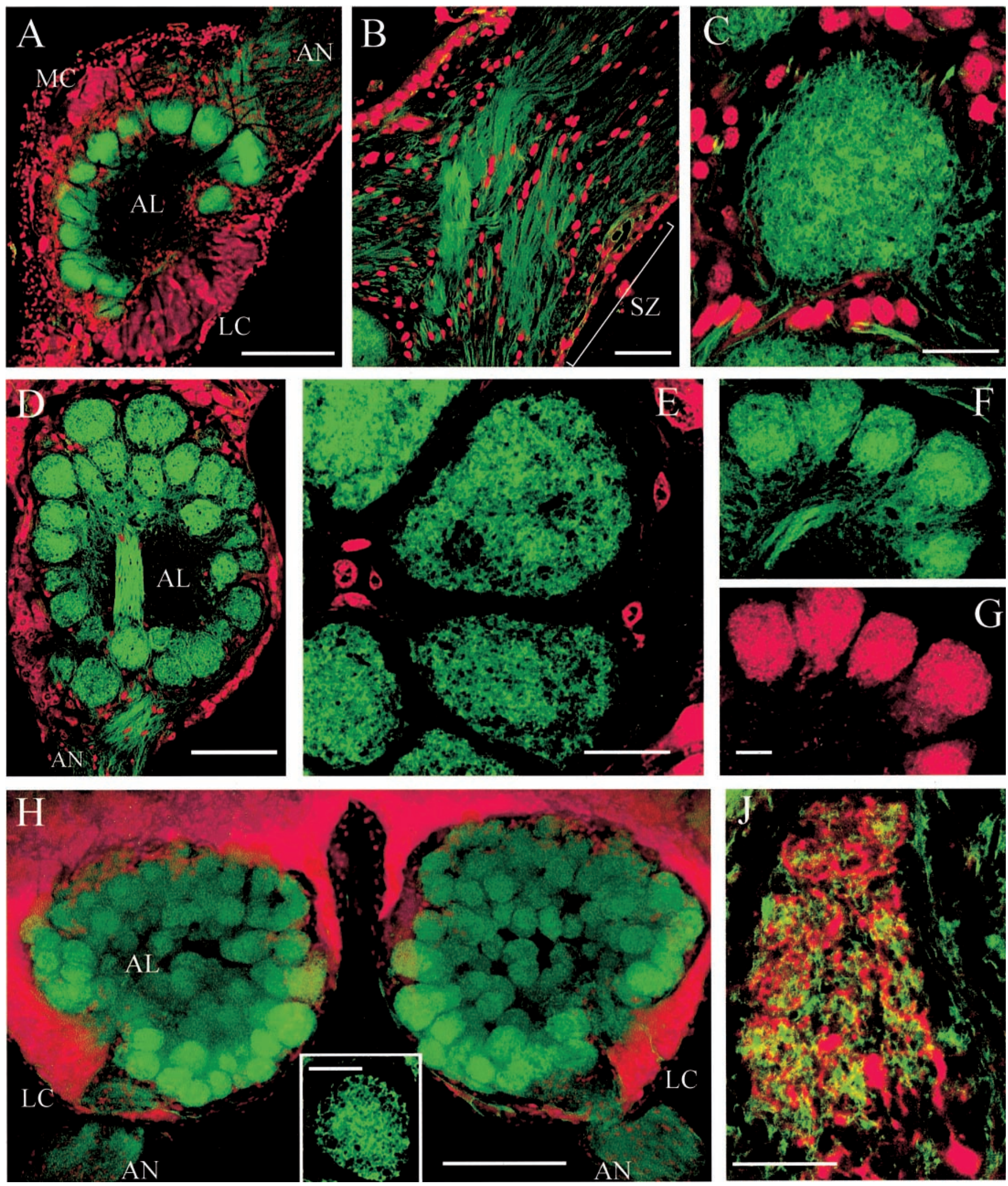


\section{Distribution of F-actin within glomeruli}

To look more closely into the compartmentalization of F-actin within the glomeruli, we performed double-labeling experiments using antibodies to the synaptic-vesicle proteins synaptophysin and synapsin. Both proteins are associated with synaptic vesicles and are restricted to presynaptic compartments (Jahn et al., 1985; Klagges et al., 1996). In the accessory bulb of Xenopus laevis (Figure 1C,D) and in the main OB of the rat (Figure $1 \mathrm{~J}, \mathrm{~K}$ ), at lower magnification, the distribution of synaptophysin- and synapsin-immunoreactivity (IR) and that of phalloidin staining exhibit an almost similar pattern. At higher magnification, however, a mismatch synaptic-protein IR and F-actin staining is visible. This is most evident in peripheral regions of the less densely packed glomeruli in the OB of $X$. laevis (Figure $1 \mathrm{M})$. This indicates that F-actin is located predominantly in postsynaptic dendritic compartments, which is further supported by the fact that in all vertebrates staining was completely absent from axons in the nerve layer.

The general distribution of F-actin in pre- and postsynaptic compartments appears to be different in insects. In contrast to vertebrates, in all insects we observed light phalloidin labeling along olfactory receptor axons within the antennal (olfactory) nerve, in addition to strong labeling within the glomeruli (Figure 2B,D,F,H). The dendritic branches and processes of neurons and glial cells within the central core of the ALs of insects were not labeled at all (Figure 2A,D,H). Axonal staining was especially prominent in an ORN-axon tract that crosses the central core of the AL in the honeybee (Figure 2D). The axonal staining pattern, as shown, for example, in Manduca sexta (Figure 2B), can be very useful in the visualization of the trajectories of axon bundles within the glia-rich receptor axon sorting zone at the entrance of the olfactory nerve (Rössler et al., 1999b). In the honeybee, synapsin-IR and phalloidin labeling show a differential localization even at lower magnification (Figure 2F,G). Synapsin was concentrated exclusively within the glomeruli whereas phalloidin labeling was also present along the axon tracts of receptor neurons. To further explore the compartmentalization of F-actin within glomeruli, we traced projection neurons (PNs) intracellularly with rhodamin-coupled dextrans and stained sections with phalloidin (Figure 2J). The example shown in the cricket demonstrates that phalloidin labeling showed only very little overlap with the dendritic branches of PNs. Phalloidin only labeled branches with very fine profiles. A partial overlap, however, with the very fine dendritic tips cannot be excluded, but in the majority of PN dendrites F-actin appears to be absent. Taken together, the results indicate that, in contrast to vertebrates, in insects F-actin appears to be localized predominantly in presynaptic axonal compartments within the glomeruli.

\section{Discussion}

Our results demonstrate that high concentration of F-actin represents a fundamental feature of olfactory glomeruli in species across a broad range of phyla. The compartmentalization of F-actin in axonal and dendritic elements, however, seems to differ in vertebrates and insects. Strong accumulation of F-actin within the glomerular synaptic neuropil indicates that the actin-based cytoskeleton may play an important role in synaptic and structural plasticity within glomeruli (Matus, 1999; Morales et al., 2000; De Camilli et al., 2001). In addition, our results show that phalloidin-labeling reveals the intraglomerular cytoarchitecture at excellent resolution and allows complete mapping of the entire glomerular array in a great variety of species. This will be especially useful in species for which specific molecular markers are not available and/or neuronal tracing techniques are difficult to apply.

Phallotoxins, isolated from the mushroom Amanita phalloides, are bicyclic peptides that bind the various isoforms of F-actin with high affinity (Wieland, 1987; Haugland, 1996). Because of their small size and low molecular weight ( $\sim 800$ daltons), phalloidin conjugates penetrate easily into the tissue, thus allowing staining of thick sections or whole mount preparations for confocal microscopy. In recent years, various efforts were made to provide a more or less complete spatial map of olfactory glomeruli within primary olfactory centers [e.g. in the honeybee Apis mellifera (Flanagan and Mercer, 1989; Galizia et al., 1999); in the fruit fly Drosophila (Laissue et al., 1990); in the moth Manduca sexta (Rospars and Hildebrand, 2000); and in the zebrafish Brachydanio rerio (Baier and Korsching, 1994)]. Series of histological sections, tracing of the olfactory nerve or immunocytochemistry tools were used to gain information about the spatial organization of glomeruli. However, in many 'non-model' species immunocytochemical markers often are not at hand. In other cases, anatomical features or the small size of animals exclude conventional tracing techniques. In addition, mass fills of axons or dendrites can be incomplete, and glomeruli may easily be missed. Phalloidin labeling therefore provides a powerful tool for spatial mapping of glomeruli, and allows future studies on the organization and structural plasticity of olfactory glomeruli.

F-actin isoforms are highly conserved across species, and in neurons, actin microfilaments are most abundant in presynaptic terminals, dendritic spines, growth cones and the subplasmalemmal cortex (Berl et al., 1973; Lee and Cleveland, 1996; Matus, 1999; Capani et al., 2001; De Camilli et al., 2001). In all species we investigated, phalloidin binds with high affinity to structures across the entire volume of individual glomeruli. The results indicate that in insects F-actin predominantly is localized in terminals of olfactory receptor axons whereas in vertebrates it is absent from axons and appears to be mostly aggregated in dendritic 
terminals of olfactory bulb neurons. To determine the precise distribution at densely packed glomerular synapses, future investigations at the ultrastructural level are needed.

F-actin was shown to play an important role during neuronal growth, and there is strong evidence for a role of the actin-based cytoskeleton in the regulation of synaptic vesicle dynamics (Morales et al., 2000; de Camilli et al., 2001). Furthermore, studies in vertebrates have shown that most ion-channel-linked glutamate receptors at spine synapses are closely associated with the actin cytoskeleton, and therefore indicate an important role of F-actin in the regulation of postsynaptic plasticity and spine dynamics [reviewed by Matus (Matus, 1999)]. A possible reason for the difference in the distribution of F-actin in axonal and dendritic compartments between insects and vertebrates may be the absence of dendritic spines in insect glomeruli. Thus, the mechanisms of actin-based synaptic and structural plasticity within glomeruli may differ between insects and vertebrates.

Aggregation of F-actin within glomeruli is very distinct, even in species that lack periglomerular cellular borders like in Xenopus laevis tadpoles, the axolotl and goldfish, resulting in a very loose appearance of the glomerular organization in histological preparations (Byrd and Burd, 1991; Meyer et al., 1996; Nezlin and Schild, 2000). Also in these cases, F-actin is clustered in a glomerular fashion and almost completely absent from interglomerular spaces, even though these spaces are occupied by bypassing axons and dendrites from other neurons.

In summary, our results demonstrate a consistently high concentration of F-actin in olfactory glomeruli across a broad range of species, indicating that this is an essential feature of olfactory glomeruli that is most likely related to a high degree of synaptic and structural plasticity within the glomerular neuropil of primary olfactory centers.

\section{Acknowledgements}

This paper was written in memory of Dr D.L. Meyer. The authors thank Gudrun Federkeil, Malu Obermayer, Mrs Knierim and Alexandra Kneissl for excellent technical assistance, and Drs E. Buchner and R. Jahn for generous supply with antibodies. This work was supported by the DFG, SFB 406, A12.

\section{References}

Astic, L. and Saucier, D. (2001) Neuronal plasticity and regeneration in the olfactory system of mammals: morphological and functional recovery following olfactory bulb deafferentation. Cell. Mol. Life Sci., 58, 538-545.

Baier, H. and Korsching, S. (1994) Olfactory glomeruli in the zebrafish form an invariant pattern and are indetifiable across animals. J. Neurosci., 14, 219-230.

Berl, S., Puszkin, S. and Nicklas W.J.K. (1973) Actomyosin-like protein in the brain. Science, 179, 441-446.

Byrd, C.A. and Burd, G.D. (1991) Development of the olfactory bulb in the clawed frog, Xenopus laevis: a morphological and quantitative analysis. J. Comp. Neurol., 314, 79-90.

Capani, F., Marton, M.E., Deerinck, T.J. and Ellisman, M.H. (2001) Selective localization of high concentrations of F-actin in subpopulations of dendritic spines in rat central nervous system: a three-dimensional electron microscopy study. J. Comp. Neurol., 435, 156-170.

De Camilli, P., Haucke, V., Takei, K. and Mugnani, E. (2001) The structure of synapses. In Cowan, M.W., Südhof, T.L. and Stevens, C.F. (eds), Synapses. Johns Hopkins University Press, Baltimore, MD, pp.89-133.

Farbman, A.I. (1994) Developmental biology of olfactory sensory neurons. Semin. Cell Biol. 5:3-10.

Flanagan, D. and Mercer, A.R. (1989) An atlas and 3-D reconstruction of the antennal lobes in the worker honey bee, Apis mellifera $L$. (Hymenoptera: Apidae). Int. J. Insect Morphol. Embryol., 18, 145-159.

Galizia, C.G., Mcllwrath S.L. and Menzel, R. (1999) A digital threedimensional atlas of the honeybee antennal lobe based on optical sections acquired by confocal microscopy. Cell Tissue Res., 295, 383-394.

Haugland, R.P. (1996) Handbook of Fluorescent Probes and Research Chemicals, 6th edn. Molecular Probes, Inc., Eugene, OR.

Hildebrand J.G. and Shepherd, G.M. (1997) Mechanisms of olfactory discrimination: converging evidence for common principles across phyla. Annu. Rev. Neurosci., 20, 595-631.

Jahn, R., Schiebler, W., Ouimet, C. and Greengard, P. (1985) A 38,000 dalton membrane protein (p38) present in synaptic vesicles. Proc. Natl Acad. Sci. USA, 82, 4137-4141.

Klagges, B.R.E., Heimbeck, G., Godenschwege, T.A., Hofbauer, A., Pflugfelder, G.O., Reifegerste, R., Reisch, D., Schaupp, M., Buchner, S. and Buchner, E. (1996) Invertebrate synapsins: a single gene codes for several isoforms in Drosophila. J. Neurosci.,16, 3154-3165.

Laissue, P.P., Reiter, C., Hiesinger, P.R., Halter, S., Fischbach, K.F. and Stocker, R.F. (1999) Three-dimensional reconstruction of the antennal lobe in Drosophila melanogaster. J. Comp. Neurol., 405, 543-552.

Lee, M.K. and Cleveland, D.W. (1996) Neuronal intermediate filaments. Annu. Rev. Neurosci., 19, 187-217.

Matus, A. (1999) Postsynaptic actin and neuronal plasticity. Curr. Opin. Neurobiol., 9, 561-565.

Meyer, D.L., Jadhao, A.G., Bhargava, S. and Kicliter, E. (1996) Bulbar representation of the 'water-nose' during Xenopus ontogeny. Neurosci. Lett., 220, 109-112.

Morales, M., Colicos, M.A. and Goda, Y. (2000) Actin-dependent regulation of neurotransmitter release at central synapses. Neuron, 27, 539-550.

Nezlin, L.P. and Schild, D. (2000) Structure of the olfactory bulb in tadpoles of Xenopus laevis. Cell Tissue Res., 302, 21-29.

Nieuwkoop, P.D. and Faber, J. (1956) Normal Table of Xenopus laevis (Daudin). Elsevier-North Holland, Amsterdam.

Oland, L.A. and Tolbert, L.P. (1996) Multiple factors shape development of olfactory glomeruli: insights from an insect model system. J. Neurobiol., 30, 92-109.

Rospars, J.P. and Hildebrand, J.G. (2000) Sexually dimorphic and isomorphic glomeruli in the antennal lobes of the sphinx moth Manduca sexta. Chem. Senses, 25, 119-129

Rössler, W., Tolbert, L.P. and Hildebrand, J.G. (1998) Early formation of 
sexually dimorphic glomeruli in the developing olfactory lobe of the brain of the moth Manduca sexta. J. Comp. Neurol., 396, 415-428.

Rössler, W., Randolph, P.W., Tolbert, L.P. and Hildebrand, J.G. (1999a) Axons of olfactory receptor cells of trans-sexually grafted antennae induce development of sexually dimorphic glomeruli in Manduca sexta. J. Neurobiol., 38, 521-541.

Rössler, W., Oland, L.A., Higgins, M.R., Hildebrand, J.G. and Tolbert, L.P. (1999b) Development of a glia-rich axon-sorting zone in the olfactory pathway of Manduca sexta. J. Neurosci., 19, 9865-9877.

Sigg, D., Thompson, C.M. and Mercer, A.M. (1997) Activity-dependent changes to the brain and behavior of the honey bee, Apis mellifera (L.). J. Neurosci., 17, 7148-7156.

Tolbert, L.P. and Hildebrand, J.G. (1981) Organization and synaptic ultrastructure of glomeruli in the antennal lobes of the moth Manduca sexta: a study using thin sections and freeze fracture. Proc. R. Soc. Lond. B, 213, 279-301.

Tolbert, L.P and Oland, L.A. (1990) Glial cells form boundaries for developing insect olfactory glomeruli. Exp. Neurol., 109, 19-28.
Treolar, H.B., Purcell, A.L. and Greer, C.A. (1999) Glomerular formation in the developing rat olfactory bulb. J. Comp. Neurol., 413, 289-304.

Valverde, F. (1999) Building an olfactory glomerulus. J. Comp. Neurol., 415, 419-422.

Wieland, T. (1987) 50 Jahre Phalloidin. Naturwissenschaften, 74, 367-373.

Winnington, A.P., Napper, R.M. and Mercer, A.R. (1996) Structural plasticity of identified glomeruli in the antennal lobes of the adult worker honey bee. J. Comp. Neurol., 365, 479-490.

Wolff J.R. and Missler, M. (1992) Synaptic reorganization in developing and adult nervous systems. Ann. Anat., 174, 393-403.

Xu, F., Greer, C.A. and Shepherd, G.M. (2000) Odor maps in the olfactory bulb. J. Comp. Neurol., 422, 489-495.

Zippel, H.P. (2000) In goldfish the discriminative ability for odours persists after reduction of the olfactory epithelium, and rapidly returns after olfactory nerve axotomy and crossing bulbs. Phil. Trans. R. Soc. B, 355, 1219-1223.

Accepted August 23, 2002 